\title{
HyperForce: Hypervisor-enForced Execution of Security-Critical Code
}

\author{
Francesco Gadaleta, Nick Nikiforakis, Jan Tobias Mühlberg, \\ and Wouter Joosen \\ IBBT-DistriNet, KU Leuven, Celestijnenlaan 200A B-3001, Leuven, Belgium \\ \{francesco.gadaleta, nick.nikiforakis, jantobias.muehlberg, \\ wouter.joosen\}@cs.kuleuven. be
}

\begin{abstract}
The sustained popularity of the cloud and cloud-related services accelerate the evolution of virtualization-enabling technologies. Modern off-the-shelf computers are already equipped with specialized hardware that enables a hypervisor to manage the simultaneous execution of multiple operating systems. Researchers have proposed security mechanisms that operate within such a hypervisor to protect the virtualized operating systems from attacks. These mechanisms improve in security over previous techniques since the defense system is no longer part of an operating system's attack surface. However, due to constant transitions between the hypervisor and the operating systems, these countermeasures typically incur a significant performance overhead.

In this paper we present HyperForce, a framework which allows the deployment of security-critical code in a way that significantly outperforms previous in-hypervisor systems while maintaining similar guarantees with respect to security and integrity. HyperForce is a hybrid system which combines the performance of an in-guest security mechanism with the security of in-hypervisor one. We evaluate our framework by using it to re-implement an invariance-based rootkit detection system and show the performance benefits of a HyperForceutilizing countermeasure.
\end{abstract}

Keywords: virtualization, hypervisor, virtual devices, countermeasure.

\section{Introduction}

The "cloud" is probably the most used technological term of the last years. Its supporters present it as a complete change in the way that companies operate that will help them scale on-demand without the hardware-shackles of the past. CPU-time, hard-disk space, bandwidth and complete virtual infrastructure can be bought at a moment's notice. Backups of data are synced to the cloud and in some extreme cases, all of a user's data may reside there (Chromium OS). Its opponents treat it as privacy nightmare that will take away the user's control over their own data and place it in the hands of corporations as well as a risk to the privacy, integrity and availability of user data [3|13. Regardless however 
on one's view of the cloud, one of the main technologies that makes the cloudconcept possible is virtualization.

Virtualization is the set of technologies that together allow for the existence of more than one running operating systems on-top of a single physical machine. While initially all of the needed mechanisms for virtualization were created in software, the sustained popularity of virtualization, lead to their implementation in hardware, providing the desired speed that was lacking in their software counterparts. Today both Inte 1 and $\mathrm{AMD}^{2}$ support a set of instructions that are there with the sole purpose of facilitating virtualization. Apart from the use of virtualization as way to host different operating systems on one machine, virtualization can also be used to provide greater security guarantees for operating systems. Researchers have already proposed various system that use virtualization primitives that all fall in this category 56678 . The chief difference between these systems that operate from within the virtualized system's hypervisor (in-hypervisor) and protection systems that operate from within the operating system (in-guest) is that the latter are part of the system's attack surface. For instance, an antivirus that operates from within the operating system that it supposedly protects, i.e. in-guest, could be deactivated or crippled by the attack itself. In-hypervisor security systems, in contrast, can utilize the isolation guarantees of virtualization to make sure that they will be active regardless of the state of system that they protect. Unfortunately these security benefits do not come for free. The constant transition from the virtualized operating system to the hypervisor that protects it (known as VMExit) and back (VMEntry), negatively affects the performance of the virtualized systems forcing one to choose between better security or better performance.

In this paper we present HyperForce, a framework that allows countermeasures for virtualized operating systems to be protected, with security and integrity comparable to the one provided by in-hypervisor systems but at the performance cost of in-guest systems. Our system follows a hybrid approach by maintaining the security-critical code within the guest but forcing its execution and protecting its instructions and data from the hypervisor. Using our framework, we re-implement Hello rootKitty [7, an in-hypervisor rootkit-detection system which uses the invariance of critical kernel objects as a way of identifying kernel compromises. We evaluate the implementation of Hello rootKitty using our framework and show that it significantly outperforms the original version while maintaining comparable security guarantees.

The rest of this paper is structured as follows. In Section 2 we present our motivation for the HyperForce framework followed by its design details. In Section 3 we evaluate the performance benefits of our framework by using it to re-implement the aforementioned rootkit-detection system. In Section 4 we discuss the related work and Section 5 concludes.

\footnotetext{
1 http://www.intel.com/technology/virtualization/technology.htm

2 http://sites.amd.com/us/business/it-solutions/virtualization
} 


\section{Design}

In this section, we describe the needs that motivated us to create HyperForce and we provide the design and implementation details of our system.

\subsection{Motivation}

Designing a countermeasure that protects virtualized operating systems is considered a challenge not only because of the difficulty to modify the target system (due to the lack of sources or licenses) but also because a virtualized system is already affected by consistent overhead, by design. An important goal for any framework using virtualization as a security tool, is to guarantee the execution of critical code in the kernel-space of a virtualized operating system regardless of the state of the kernel, i.e. code that will run identically in both clean and compromised kernels. By critical code we refer to code that, in general, monitors the state of the system and that it is desirable, mainly from a security point of view, to maintain its execution. Examples of such code include the integrity check of sensitive kernel-level data structures that are usually abused by rootkits or the scanning of files and memory for known malware signatures. Given our assumption of a kernel-level attacker, it is also needed to ensure the integrity of the critical code to protect it from malicious modifications which might compromise its efficacy or completely disable its operations.

A straightforward way of achieving this goal is to implement and execute security-critical code within the hypervisor [7]. An alternative approach monitors the target system from a separate virtual machine. In fact, one of the most interesting features of virtualization technology is that it guarantees complete isolation between the hypervisor and any virtual machine running on top of it as well as isolation between multiple virtual machines running on top of the same physical machine. Unfortunately, both approaches are known to be affected by consistent performance overhead, making it hard to consider such solutions for production systems. The main goal of HyperForce is to keep a degree of security comparable to these completely isolated systems while significantly reducing their performance overhead.

\subsection{Core Idea}

The idea of HyperForce is to combine the best features of the in-guest and in-hypervisor defense systems into a hybrid solution which performs as an in-guest countermeasure while providing security comparable to in-hypervisor countermeasures. We achieve this by deploying the functional part of the countermeasure within the guest operating system while maintaining its integrity and enforcing its execution with the assistance of the hypervisor. Since the functional part of the security-critical code, i.e. its instructions and data, is running within the virtualized operating system, it also has native access to the resources of the virtualized operating system such as the memory, disk and API of the virtualized kernel. This provides a great performance benefit for code that 
needs to access many memory locations within the virtualized operating system since it alleviates the costly need of introspection that in-hypervisor systems require, i.e. the discovery of the corresponding physical memory pages of the virtual memory pages of the guest and their remapping within the hypervisor or within another virtual machine.

Enforcement of Execution. Given an arbitrary piece of security-critical code, HyperForce needs to ensure its execution at regular time intervals. A complete reliance for its execution on the virtualized operating system, could potentially allow a kernel-level attacker to intervene and inhibit the code's execution through the modification of the appropriate kernel-level data-structures. For instance, an attacker could locate the function pointer pointing to the security-critical code and overwrite it with a pointer towards their own code.

From a high-level view, HyperForce changes the execution flow of the guest kernel whenever the installed monitoring code has to be executed and restores the original execution flow upon code termination. The advances of virtualization technology allows one to implement this transition in a multitude of ways. Our decision was influenced by our desire of minimizing the amount of instrumentation code in the hypervisor and of keeping performance overhead to a minimum.

In our framework, the security-critical code is encapsulated within a function that is loaded in the virtualized operating system in the form of Linux Kernel Module (LKM). This allows the code to have native access to all of the VM's native resources. HyperForce then uses the infrastructure of the virtualization platform, specifically the Virtual Machine Monitor (VMM), to create a virtual device. Virtual devices simulate real hardware devices, such as sound-cards and video cards, and are supported by all modern Virtual Machine Monitors. Once this virtual device is created and loaded in the virtualized operating system, HyperForce then registers the address of the security-critical code as an interrupt handler for the virtual device, as illustrated in Figure 1. The cooperation of the hypervisor and the trusted module, allows for the security-critical code to execute every time that the virtual device generates an interrupt.

Since the virtual device is fully controlled by the hypervisor, it is the hypervisor that decides when interrupts must be generated and not the virtualized operating system. Due to this fact, the possibly compromised kernel of the VM, cannot anticipate when the security-critical code will be executed since the logic behind it is hidden from it through the virtualization-guaranteed isolation between hypervisor and VM. This fact stops any attackers' efforts to evade detection by mimicking a non-compromised operating system just before the execution of the critical-code and restoring their malicious activities after it.

Integrity of Code. In the previous paragraphs we described the loading of security-critical code within the hypervisor and the use of virtual devices to ensure the execution of that code. Since the code is loaded in the VM as a LKM, it executes with the privileges of the virtualized kernel. While this is 


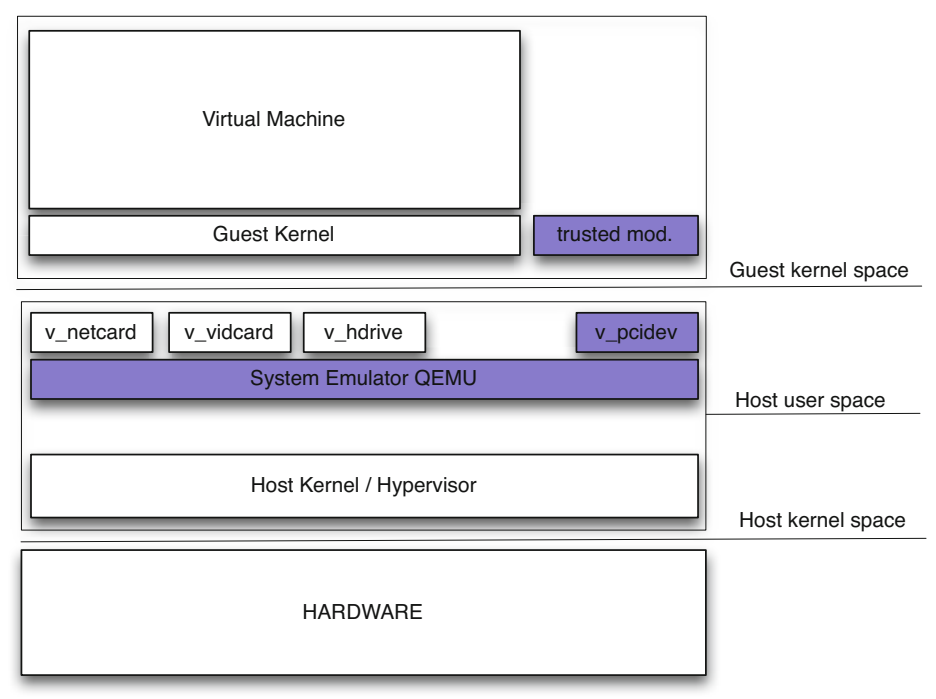

Fig. 1. Schema of HyperForce. Highlighted components indicate parts of the system that need instrumentation/modification.

desired, it also opens up the code to attacks, e.g. modifications of its code and data, from an attacker who is in control of the virtualized kernel. Traditionally, the module could not be protected from the rest of the kernel since they both operate within the same protection ring, namely Ring 0 . Due to virtualization however, the hypervisor has more power than the virtualized operating system's kernel (signified as Ring -1) and can thus protect any resources from the virtualized kernel, including memory pages. HyperForce takes advantage of this fact, and write-protects the memory pages holding the instructions and data of the security-critical code. In order to allow the code to make changes to its data, HyperForce can unlock the memory pages before it triggers an interrupt of its virtual device and lock them back immediately after the code's execution. In order to ensure that an attacker cannot avoid the execution of the interrupt handler containing the security-critical code, HyperForce also write-protects the memory page holding the Interrupt Descriptor Table (IDT) of the protected VM. Lastly, HyperForce protects the Interrupt Descriptor Table Register (IDTR) that contains the address of the IDT, as a regular invariant critical kernel object.

\section{Evaluation}

We implemented HyperForce in KVM, an extension of the Linux kernel with hypervisor capabilities. KVM is formed by a system emulator, QEMU, that runs as regular process in user space and a kernel-space device driver that uses virtualization-enabled processors. In order to show the improvements provided 
by our framework we chose to re-implement and measure a pure in-hypervisor countermeasure, namely Hello rootKitty [7].

Hello rootKitty is a lightweight invariance-enforcing framework that mitigates the problem of kernel-level rootkits. It represents a typical in-hypervisor monitoring system that checks the integrity of invariant guest-kernel objects from the hypervisor. A periodical mapping of guest-kernel memory into hypervisor space is followed by computation of its hash and checks against a set of precomputed values. Such a countermeasure often deals with a high number of kernel objects and performance overhead can easily make the guest system unusable. To minimize the amount of time spent by additional code, only a subset of these objects is checked whenever control returns to hypervisor (VMExit). Thus a certain number of VMExit events is needed to check the entire list of protected objects. This relaxation will have a cost in terms of detection time needed to check the entire list of objects.

The original version of Hello rootKitty was implemented in BitVisor [19], a tiny hypervisor designed for mediating I/O access from a single guest operating system. In order to be able to fairly compare it with our implemented version using HyperForce, we also re-implemented the original Hello rootKitty in KVM. A schema of Hello rootKitty implemented in KVM is provided in Fig. 2. It can be observed that while the HyperForce framework requires only the system emulator to be modified (Fig. 1), the implementation of the in-hypervisor Hello rootKitty needs instrumentation code to be added to the host kernel. In both cases the trusted module needs to be added to the guest kernel.

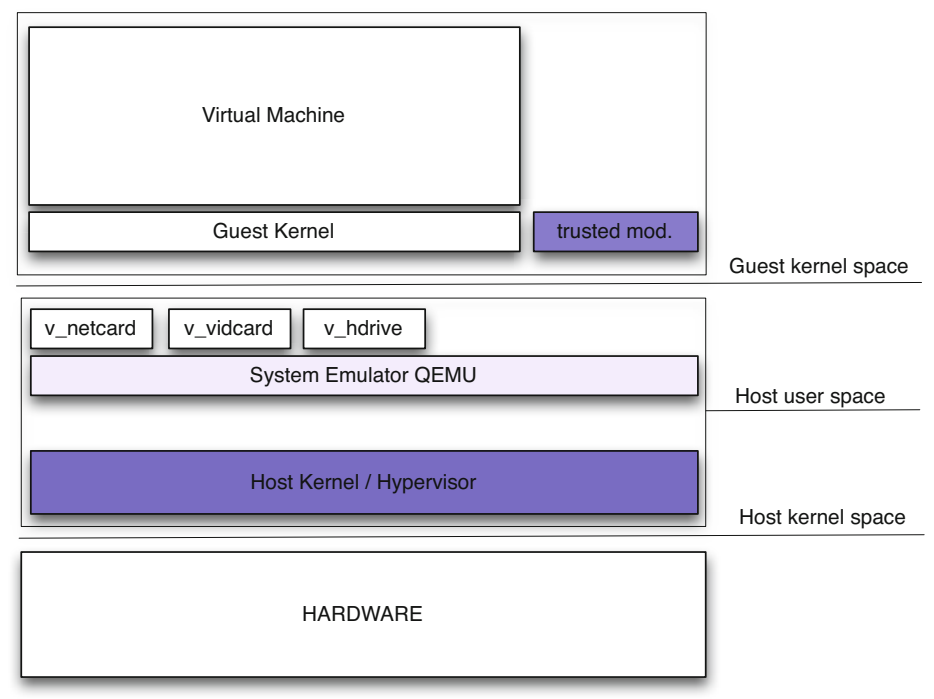

Fig. 2. Schema of Hello rootKitty implemented in Linux KVM. Highlighted components indicate parts of the system that need instrumentation/modification. 
Table 1. Macro benchmarks (in-host OS and in-guest OS) evaluating Hello rootKitty implemented with and without HyperForce

(a) In-host measurements

\begin{tabular}{|l|l|l|}
\hline & iperf [Gb/s] & overhead \\
\hline HRK & 6.36 & \\
HF(HRK) & 6.29 & $+1.1 \%$ \\
\hline
\end{tabular}

(b) In-guest measurements

\begin{tabular}{|l|l|l|}
\hline & iperf [Gb/s] & bunzip [sec] \\
\hline native KVM & 5.97 & 32.04 \\
\hline HRK & $5.26(+12 \%)$ & $33.73(+5 \%)$ \\
HF $($ HRK $)$ & $5.71(+4.3 \%)$ & $32.88(+2.5 \%)$ \\
\hline
\end{tabular}

We collected results of macro and micro-benchmarks from the guest and from the host machine and discuss them in Section 3.1 and Section [3.2. In order to provide reliable results, all tests have been repeated 10 times and averaged. Experiments have been performed on Intel Core 2 Duo 2 Ghz processor with $4 \mathrm{~GB}$ of RAM.

\subsection{Macro-benchmarks}

We run two macro benchmarks, iperf that measures TCP and UDP bandwidth performance and bunzip of a Linux kernel source code. The original in-hypervisor version of Hello rootKitty is denoted as "HRK" while the version using the HyperForce framework is denoted as "HF(HRK)".

While the in-hypervisor approach, due to the slower context switching, has a slightly better throughput of network performance in the host machine Table 1(a), benchmarks in the guest machine show a considerably better performance with HyperForce. iperf and bunzip have also been executed on a native KVM system and compared against the same system running inhypervisor Hello rootKitty and then HF(HRK). The performance overhead of our approach is about half of the in-hypervisor Hello rootKitty, as shown in Table 1(b). Hello rootKitty implemented using HyperForce performs with 4.3\% overhead compared to a native KVM guest while in-hypervisor Hello rootKitty shows $12 \%$ overhead. The second column reports overhead of bunzip measured in seconds. $\mathrm{HF}(\mathrm{HRK})$ outperforms the in-hypervisor Hello rootKitty, showing an overhead of only $2.5 \%$ compared to the native KVM guest.

\subsection{Micro-benchmarks}

Micro benchmarks show a more detailed picture of the two approaches. We use LMbench $[12]^{3}$ to measure the overhead of operating system specific events such as context switch, memory mapping latency, page fault, signal handling and fork. Within the host machine, HyperForce shows substantial improvement against the alternative Hello rootKitty. In Table 2 we report only the tests where this improvement is consistent. In all other tests the in-hypervisor Hello rootKitty and the Hello rootKitty using HyperForce show negligible performance overhead.

\footnotetext{
${ }^{3}$ We use version 3 of LMbench as available at http://lmbench.sourceforge.net/
} 
Table 2. Overhead of Hello rootKitty using the HyperForce framework (HF(HRK)) is measured against in-hypervisor Hello rootKitty (HRK) with LMbench microbenchmarks within the host machine. Operations are measured in microseconds.

\begin{tabular}{|l|c|c|c|c|}
\hline & ctx switch & mmap lat & page flt & mem lat \\
\hline HRK & 2.020 & 6148 & 1.57 & 114.7 \\
HF(HRK) & 1.48 & 4950 & 1.46 & 101.7 \\
\hline speedup & $+26 \%$ & $+19 \%$ & $+7 \%$ & $+11 \%$ \\
\hline
\end{tabular}

Table 3. Overhead of $\mathrm{HF}(\mathrm{HRK})$ is measured against in-hypervisor Hello rootKitty with LMbench micro-benchmarks within the guest machine. Operations are measured in microseconds.

\begin{tabular}{|l|c|c|c|c|c|c|c|c|}
\hline & null call & null IO & open/close & sig inst & sig handl & fork proc & exec proc & ctx switch \\
\hline HRK & 0.30 & 0.32 & 2.32 & 0.74 & 5.37 & 1923 & 4087 & 5.58 \\
HF(HRK) & 0.14 & 0.21 & 2.10 & 0.45 & 2.60 & 1788 & 3984 & 5.00 \\
\hline \hline Speedup & $+53 \%$ & $+34 \%$ & $+10 \%$ & $+39 \%$ & $+51 \%$ & $+8 \%$ & $+2.5 \%$ & $+10 \%$ \\
\hline
\end{tabular}

The picture in the guest machine shows a similar trend in which $\mathrm{HF}(\mathrm{HRK})$ outperforms the original in-hypervisor Hello rootKitty in every test (Table 3).

To interpret the results shown in Table 3, one has to know that Hello rootKitty performs integrity checks whenever the guest kernel writes to a control register (MOV_CR* event). When virtual addressing is enabled, the upper 20 bits of control register 3 (CR3) become the page directory base register, which is used to locate the page directory and page table of the current process. Thus, on every context switch or system call invocation, CR3 is modified. Trapping these events strategically contributes to Hello rootKitty's short attack detection time. Yet, the integrity checks performed by Hello rootKitty increase the latency of context switches and system calls.

In contrast, our implementation of Hello rootKitty in HyperForce employs interrupt events to trigger in-guest integrity checks. This eliminates overheads with respect to switching execution context and address mapping between the hypervisor and the guest OS, while the remaining computational overhead affects guest operations more evenly. As can be seen in Table 3. the above changes imply significant speedups on system call invocations (53\%) and context switches (10\%). Although LMbench is often considered as insufficient for evaluating system performance [10, our example shows that the benchmark suite can be used to neatly distinguish the actual speedup on system call invocations ("null call") from the impact on a particular system call execution (e.g. "open/close").

One may think that our approach to trigger security checks through interrupts in HyperForce reduces the security of the protected system compared to the original in-hypervisor Hello rootKitty: in the latter case an attacker increases their chance of being detected with every system call raised. However for a total 
of 15,000 protected kernel objects, the worst-case detection time reported in [7] is 6 seconds. Hello rootKitty in HyperForce improves on that by checking the same amount of kernel objects in 4 seconds. While Hello rootKitty relies on the activity of the system as a trigger that checks the integrity of protected objects, Hello rootKitty in HyperForce performs the checking independently of system activity every 4 seconds.

In summary, our implementation of Hello rootKitty in HyperForce significantly reduces computational overhead while reducing the worst-case detection time for potentially malicious manipulations of invariant kernel objects. Our results indicate that the HyperForce framework could be used to re-implement other in-hypervisor applications, enhancing their performance and maintaining their effectiveness.

\section{Related Work}

In this section we review related work in the domain of kernel code integrity assurance. For a discussion of literature related to rootkit detection we refer the reader to [7].

Hardware-Based Execution Flow Integrity. Means of guaranteeing the integrity of a running operating system that employ dedicated hardware devices to monitor the physical memory of a computer system have been proposed in [1] and [14. In order to perform integrity checks, both systems make use of PCI hardware that directly accesses the computer's memory at a negligible performance overhead. Yet, the need for dedicated hardware may hinder widespread deployment of these techniques.

Hypervisor-Based Execution Flow Integrity. A tiny hypervisor that protects legacy OSs by ensuring that only validated code can be executed in kernel mode, is SecVisor [17. A similar system, NICKLE [16], shadows physical memory to store authenticated guest code. At runtime, an instruction fetch is directed to access either the normal system memory or the shadow area, depending on whether the instruction is to be executed in user mode or kernel mode. An attempt to execute unvalidated code can thus be detected and prevented. Recently, attacks that do not inject malicious code but construct it from existing fragments of the attacked program have been presented [2918]. These attacks effectively bypass countermeasures such as SecVisor and NICKLE.

Rootkits commonly modify a system's function pointers to ensure execution. HookScout 21] detects such rootkits. The tool employs as system emulator to infer a policy for function pointer propagation in kernel memory. A separate detection system is then used to detect violations of this policy during normal operation of the OS. Since the detection system runs on the target machine, it may be disabled by an attack. HookSafe [20] protects kernel hooks that are dynamically allocated by relocating these kernel hooks to dedicated memory pages. Regular page-level protection through the hardware's Memory 
Management Unit is then used to protect the pages. Yet, the technique does not prevent non-control data from being compromised.

HyperForce can be utilized to effectively protect the integrity of the in-guest components of systems such as HookScout and HookSafe. Our experimental results obtained from implementing Hello rootKitty [7] in HyperForce show that our technique leverages the use of in-guest protection mechanisms. That is, HyperForce substantially reduces the performance overhead that would occur if the countermeasure would be implemented in-hypervisor, while strong security guarantees are maintained.

Security Agent Injection. Closely related to HyperForce is work by Lee et al. 11] and Chiueh et al. 4] on deploying agents by means of code injection from a hypervisor. Both approaches are applicable to guest OSs that have not been previously prepared by loading a special driver or similar. In [11], Lee et al. proposes to protect agent code that is executing in a compromised guest OS kernel by the use of cryptography and by injecting this code on demand from the hypervisor. As there is no implementation and no experimental evaluation given, a comparison with HyperForce is not feasible. Similarly, work on SADE 4 by Chiueh et al. uses VMWare's ESX server API to inject and execute code in a guest OS so as to disable and remove a previously detected malware infection from that guest. In difference to the HyperForce approach, the agent code in SADE is not protected from malicious interference on the guest. Chiueh et al. argue that on-demand injection leaves a relatively short time span for such interference. SADE is used by a virtual appliance that implements out-ofguest monitoring of VMs' memory, scanning for malware signatures. The paper presents experimental data on the code injection process but does not discuss the overhead implied by mapping memory pages between the virtual appliance and the VMs. We expect in-guest memory inspection, as implemented by our Hello rootKitty in HyperForce, to outperform SADE.

\section{Conclusion}

The attractive properties offered by virtualization are a foundational block for the whole "cloud technology". At the same time, virtualization is already being used for purposes other than the deployment of multiple operating systems as a way of increasing the security of a single virtualized operating system. In this paper we briefly discuss the differences between security mechanisms deployed within an operating system (in-guest) and the ones deployed within a hypervisor (in-hypervisor) and bring attention to the, seemingly exclusive, choice between the performance benefits of the former versus the security benefits of the latter. We tackle this choice by developing HyperForce, a hybrid framework allowing security mechanisms to be developed in a way that provides them with performance analogous to in-guest systems while maintaining the security of in-hypervisor systems. Using HyperForce, we re-implemented an in-hypervisor rootkit detection system and show how the new version significantly outperforms 
the original without compromising the security or integrity of the detection system.

We conclude that hybrid security systems that are built on top of HyperForce can provide effective and efficient alternatives to mitigate the overhead of techniques that exclusively operate in-hypervisor. Interesting candidate applications for our framework are, e.g., malware detection and removal software. For future work we envisage to extend HyperForce with techniques to inject security agents into a guest operating system so as to provide secure means of on-demand deployment of such agents.

Acknowledgments. This research is partially funded by the Interuniversity Attraction Poles Programme Belgian State, Belgian Science Policy, the Research Fund KU Leuven and the EU FP7 project NESSoS.

\section{References}

1. Baliga, A., Ganapathy, V., Iftode, L.: Detecting kernel-level rootkits using data structure invariants. IEEE Transactions on Dependable and Secure Computing 8, 670-684 (2011)

2. Buchanan, E., Roemer, R., Shacham, H., Savage, S.: When good instructions go bad: generalizing return-oriented programming to RISC. In: CCS 2008: Proceedings of the 15th ACM Conference on Computer and Communications Security, pp. 2738. ACM (2008)

3. Business Insider. Amazon's Cloud Crash Disaster Permanently Destroyed Many Customers' Data (2011), http://articles.businessinsider.com/201104-28/tech/29958976_1_amazon-customer-customers-data-data-loss

4. Chiueh, T.C., Conover, M., Lu, M., Montague, B.: Stealthy deployment and execution of in-guest kernel agents. In: Proceedings of the Black Hat USA Security Conference (2009)

5. Criswell, J., Lenharth, A., Dhurjati, D., Adve, V.: Secure Virtual Architecture: A Safe Execution Environment for Commodity Operating Systems. In: SOSP 2007: Proceedings of the 21st ACM Symposium on Operating Systems Principles, pp. 351-366. ACM (2007)

6. Dewan, P., Durham, D., Khosravi, H., Long, M., Nagabhushan, G.: A hypervisorbased system for protecting software runtime memory and persistent storage. In: SpringSim 2008: Proceedings of the 2008 Spring Simulation Multiconference, pp. 828-835. Society for Computer Simulation International (2008)

7. Gadaleta, F., Nikiforakis, N., Younan, Y., Joosen, W.: Hello rootKitty: A Lightweight Invariance-Enforcing Framework. In: Lai, X., Zhou, J., Li, H. (eds.) ISC 2011. LNCS, vol. 7001, pp. 213-228. Springer, Heidelberg (2011)

8. Gadaleta, F., Younan, Y., Jacobs, B., Joosen, W., De Neve, E., Beosier, N.: Instruction-level countermeasures against stack-based buffer overflow attacks. In: Eurosys, pp. 7-12. ACM (2009)

9. Hund, R., Holz, T., Freiling, F.C.: Return-oriented rootkits: Bypassing kernel code integrity protection mechanisms. In: SSYM 2009: Proceedings of the 18th Conference on USENIX Security Symposium, pp. 383-398. USENIX Association (2009) 
10. Open Kernel labs. Why lmbench is evil?, http://www.ok-labs.com/blog/entry/why-lmbench-is-evil/

11. Lee, Y.-C., Rahimi, S., Harvey, S.: A pre-kernel agent platform for security assurance. In: IEEE Symposium on Intelligent Agent (IA), pp. 1-7. IEEE (2011)

12. McVoy, L., Staelin, C.: LMbench: Portable tools for performance analysis. In: Proceedings of the 1996 Annual Conference on USENIX Annual Technical Conference, pp. 23-39. USENIX Association, Berkeley (1996)

13. Nikiforakis, N., Balduzzi, M., Van Acker, S., Joosen, W., Balzarotti, D.: Exposing the lack of privacy in file hosting services. In: Proceedings of the 4th USENIX conference on Large-Scale Exploits and Emergent Threats, LEET (2011)

14. Petroni Jr., N.L., Fraser, T., Molina, J., Arbaugh, W.A.: Copilot - a coprocessorbased kernel runtime integrity monitor. In: Proceedings of the 13th USENIX Security Symposium, p. 13. USENIX Association (2004)

15. QubesOS: Architecture Specification, http://qubes-os.org/files/doc/arch-spec-0.3.pdf

16. Riley, R., Jiang, X., Xu, D.: Guest-Transparent Prevention of Kernel Rootkits with VMM-Based Memory Shadowing. In: Lippmann, R., Kirda, E., Trachtenberg, A. (eds.) RAID 2008. LNCS, vol. 5230, pp. 1-20. Springer, Heidelberg (2008)

17. Seshadri, A., Luk, M., Qu, N., Perrig, A.: SecVisor: a tiny hypervisor to provide lifetime kernel code integrity for commodity OSes. In: Proceedings of Twenty-First ACM SIGOPS Symposium on Operating Systems Principles, pp. 335-350. ACM (2007)

18. Shacham, H.: The geometry of innocent flesh on the bone: return-into-libc without function calls (on the x86). In: CCS 2007: Proceedings of the 14th ACM Conference on Computer and Communications Security, pp. 552-561. ACM (2007)

19. Shinagawa, T., Eiraku, H., Tanimoto, K., Omote, K., Hasegawa, S., Horie, T., Hirano, M., Kourai, K., Oyama, Y., Kawai, E., Kono, K., Chiba, S., Shinjo, Y., Kato, K.: BitVisor: a thin hypervisor for enforcing I/O device security. In: VEE 2009: Proceedings of the 2009 ACM SIGPLAN/SIGOPS International Conference on Virtual Execution Environments, pp. 121-130. ACM (2009)

20. Wang, Z., Jiang, X., Cui, W., Ning, P.: Countering kernel rootkits with lightweight hook protection. In: CCS 2009: Proceedings of the 16th ACM Conference on Computer and Communications Security, pp. 545-554. ACM (2009)

21. Yin, H., Poosankam, P., Hanna, S., Song, D.: HookScout: Proactive Binary-Centric Hook Detection. In: Kreibich, C., Jahnke, M. (eds.) DIMVA 2010. LNCS, vol. 6201, pp. 1-20. Springer, Heidelberg (2010) 